\title{
Lupus podocytopathy: a newly emerging nonimmune complex mediated nephropathy associated with systemic lupus erythematosus
}

\author{
Jinil Yoo ${ }^{1}$, Donald Baumstein ${ }^{2}$ \\ ${ }^{1}$ Division of Nephrology, Montefiore Medical Center - Albert Einstein College of Medicine, Bronx, NY, USA; ${ }^{2}$ Division of Nephrology, \\ Metropolitan Hospital Center - New York Medical College, New York, NY, USA \\ Correspondence to: Jinil Yoo, MD. Division of Nephrology, Montefiore Medical Center - Wakefield Campus, 600 East $233^{\text {rd }}$ Street, Bronx, NY 10466, \\ USA. Email: jiyoo@montefiore.org. \\ Provenance and Peer Review: This article was commissioned by the Editorial Office, Annals of Palliative Medicine. The article did not undergo external \\ peer review. \\ Comment on: Wu J, Zeng F, Jiang X, et al. Lupus podocytopathy complicated with multiple organ injuries in a patient with severe lupus. Ann Palliat \\ Med 2019;8:763-8.
}

Submitted Dec 13, 2019. Accepted for publication Jan 08, 2020.

doi: $10.21037 /$ apm.2020.01.13

View this article at: http://dx.doi.org/10.21037/apm.2020.01.13

In the article published in the November 2019 issue of Annals of Palliative Medicine, entitled "Lupus podocytopathy complicated with multiorgan injuries in a patient with severe lupus", Wu et al. (1), describes a case presenting with a fulminant form of systemic lupus erythematosus (SLE), complicated by multiple organ injuries, who was found to have acute kidney injury, 4+ proteinuria, 3+ blood on urinalysis, high titers of ANA and anti-ds DNA, and low serum complement levels. After initiation of immunosuppressive therapy to stabilize life threatening multiorgan failures, a renal biopsy was performed 3 weeks later which revealed histologic features compatible with lupus podocytopathy (LP), a newly emerging entity of nonimmune complex-mediated lupus nephropathy: in this case, no endocapillary proliferation on light microscopy (LM), negative immunofluorescence (IF), no immune complexes and no apparent thickening of glomerular basement membrane, but segmental fusion of podocyte foot processes on electron microscopy (EM). The patient was treated successfully with short-term immunosuppressive therapy. The authors explain that segmental fusion of foot processes found in their case, rather than extensive foot process effacement (FPE) on EM characteristically seen in LP might be due to intensive immunosuppressive treatments.

Lupus nephritis (LN), a common and serious complication of SLE has been considered to be a typical immune-complex mediated kidney disease and currently is classified, primarily based on histological findings of glomerular involvement, even though other structures of the kidney are involved. The 2003 ISN/RPS classification of LN (6 classes) based on histologic findings on LM and IF has been accepted worldwide for diagnosis, prognosis and treatment guidance. Class I: minimal mesangial LN with normal glomeruli on LM, but mesangial immune deposits on IF and with excellent renal prognosis. Class II: mesangial proliferative LN with mesangial hypercellularity on LM. Class III: focal proliferative and Class IV: diffuse proliferative LN, both with endocapillary or extra capillary proliferative glomerulonephritis on LM and mesangial/endothelial immune deposits on IF, requiring immunosuppressive therapy. Class V: membranous LN with glomerular basement membrane thickening and subepithelial/mesangial immune deposits. Class VI: advanced sclerotic $\mathrm{LN}$ with $>90 \%$ of glomeruli globally sclerosed without residual disease activity. The entity of LP is not included in the updated 2018 ISN/ RPS classification of $\mathrm{LN}$ yet.

Recently a subset of SLE patients presented with nephrotic syndrome (NS) in association mostly with active SLE and have been found to have predominantly FPE of podocytes on EM, but no endocapillary proliferation/ inflammation on LM, (unlike Class III and IV LN), and no glomerular capillary immune deposits on IF (unlike Class V 
LN). This subset of patients on kidney biopsy show some with minimal change disease (MCD)-like findings, some with mesangial proliferative features and some with focal segmental glomerulosclerosis (FSGS) changes, but all with diffuse FPE on EM (unlike Class I and II LN), suggestive of podocyte (visceral epithelial cell of the glomerular capillary wall) injury caused by a nonimmune complex-mediated pathway (2). Rare case reports of MCD in patients with SLE appeared in 1980s' Japanese literature and in 1990s' English literature (3).

In 2002, Dube et al. (4) reported 7 patients with SLE and clinical and pathologic features of MCD, and described the entity as an underrecognized, highly reversible form of NS, and cautioned against interpreting this as an atypical presentation of Class II LN for 5 of those 7 patients with mild mesangial proliferation. In 2005, Kraft et al. (2) described 8 SLE patients with nephrotic range proteinuria and diffuse FPE on EM, but no immune deposits in glomerular capillaries, nor endocapillary proliferation of glomeruli, and called it "LP". In 2016, Hu et al. (5) reported 50 cases of LP from 3,750 kidney biopsies of SLE patients: MCD in 13 cases, mesangial proliferation in 28 cases and FSGS in 9 cases. Clinical features based on $\mathrm{Hu}$ et al. (5) series (the largest among reported series) include NS in all patients (50 out of 50 patients), AKI in 17 patients $(34 \%)$ with 5 patients requiring renal replacement therapy and low serum complement $\left(\mathrm{C}_{3}\right)$ level in 34 patients (68\%). The clinical outcome was much more favorable to immunosuppressive therapy for the patients with MCD-like and mesangial proliferation than for patients with FSGS features. Following the induction therapy with glucocorticoids only or glucocorticoids plus other immunosuppressive agents, the patients with LP in Hu et al.'s series (5) achieved overall remission rate of $94 \%$ (complete remission in $76 \%$ ) with median time to remission in 4 weeks, even though the FSGS group showed much lower complete remission rate and longer time to remission than MCD or MP group. In all groups of LP, a high rate of relapse has been reported $(4,5)$.

In the treatment of LP, glucocorticoids are considered to be the first line therapeutic agent and other immunosuppressives to be added in relapsing cases and/ or less glucocorticoid-responsive cases of LP with FSGS features (6). Utilization of calcineurin inhibitors (CNIs) is suggested for the patients with frequent relapses since CNIs have stabilizing effects on podocyte injury (7).

The case described in the Wu et al.'s article (1) received the initial intensive immunosuppressive therapy including
IV pulse methylprednisolone (MP) and IV gamma globulin, and plasma exchange for stabilization of life threatening fulminant SLE, followed by oral MP and IV cyclophosphamide. In 4-week's therapy the patient had negative protein in the urine and resolved acute kidney injury.

In LP the absence of immune deposits in glomerular capillary walls and the presence of severe FPE of podocytes support a similar mechanism speculated for MCD or primary FSGS, likely podocyte injury mediated by the production of cytokines, lymphokines or T-cell dysfunction, independent of typical immune complex mediated injury (6).

Podocytes are highly differentiated epithelial cells of the glomerular capillary wall that when injured lead to disruption of the slit diaphragm (connecting foot processes of podocytes) which forms the main barrier to prevent proteinuria. A recent study reports that podocyte injury is associated with overexpression of angiopoietin like-4 which can induce a proteinuric response (8). In $\mathrm{LN}$, podocytes appear not only subject to injury due to glomerular capillary lesions secondary to immune complex-induced and inflammatory processes, but also, can be a direct target (9), although the exact mechanism causing LP remains unclear. There is growing evidence that glomerular podocytes are immunologically active cells. B7-1 involved in T-cell costimulation is found to be upregulated on podocytes by lipopolysaccharide (LPS) via TLR-4 activation (10). Podocytes also act as antigen presenting cells, participating in immune-mediated glomerular diseases (11).

In LN transformations from one to another class of immune complex induced glomerular lesions are often found on serial kidney biopsies. There are also documented transformations between non-immune complex mediated LP and immune-complex forms of LN. In $\mathrm{Hu}$ et al.'s series (5), 13 relapsed patients out of 50 patients with LP had a second kidney biopsy. Six of 13 patients biopsied transitioned to an immune complex mediated form of $\mathrm{LN}$, three to class IV LN, and three to Class V LN. There are also case reports of LP developing after successful treatment of immune complex-mediated LN; one case of Class III LN (focal proliferative) developed NS 5 years later and the repeat biopsy showed histologic features of MCD (12), and another case of Class V LN (membranous) had recurrent NS 6 years later and the repeat biopsy revealed histologic findings of MCD, a non-immune complex LP along with features of healed Class V (13).

In the case reported in this journal, Wu et al. describes a patient with likely LP, a non- immune complex-mediated 
form, from the patient who has a fulminant form of SLE, resulting in multiorgan failure, presumably due to full blown immune complex-mediated injury. This case report adds to our growing awareness of the entity "LP".

Even though the pathogenic mechanism is not well delineated and not accepted as a class of lupus nephropathy yet, the entity of LP is emerging as a distinct entity (14) with distinct response to treatment. It is less likely a mere association with SLE, but more likely a manifestation of an active LN since LP usually occurs concurrently with extra renal and serologic activity, and mostly within six months of SLE onset.

\section{Acknowledgments}

Funding: None.

\section{Footnote}

Conflicts of Interest: The authors have no conflicts of interest to declare.

Ethical Statement: The authors are accountable for all aspects of the work in ensuring that questions related to the accuracy or integrity of any part of the work are appropriately investigated and resolved.

Open Access Statement: This is an Open Access article distributed in accordance with the Creative Commons Attribution-NonCommercial-NoDerivs 4.0 International License (CC BY-NC-ND 4.0), which permits the noncommercial replication and distribution of the article with the strict proviso that no changes or edits are made and the original work is properly cited (including links to both the formal publication through the relevant DOI and the license). See: https://creativecommons.org/licenses/by-nc-nd/4.0/.

\section{References}

1. Wu J, Zeng F, Jiang X, et al. Lupus podocytopathy complicated with multiple organ injuries in a patient with severe lupus. Ann Palliat Med 2019;8:763-8.

Cite this article as: Yoo J, Baumstein D. Lupus podocytopathy: a newly emerging nonimmune complex mediated nephropathy associated with systemic lupus erythematosus. Ann Palliat Med 2020;9(2):126-128. doi: 10.21037/apm.2020.01.13
2. Kraft SW, Schwartz MM, Korbet SM, et al. Glomerular podocytopathy in patients with systemic lupus erythematosus. J Am Soc Nephrol 2005;16:175-9.

3. Desai N, Cimbaluk D, Lewis EJ, et al. Proteinuria in membranous lupus nephritis: the pathology is in the podocyte. Lupus 2013;22:461-8.

4. Dube GK, Markowitz GS, Radhakrishnan J, et al. Minimal change disease in systemic lupus erythematosus. Clin Nephrol 2002;57:120-6.

5. Hu W, Chen Y, Wang S, et al. Clinical-Morphological Features and Outcomes of Lupus Podocytopathy. Clin J Am Soc Nephrol 2016;11:585-92.

6. Oliva-Damaso N, Payan J, Oliva-Damaso E, et al. Lupus Podocytopathy: An Overview. Adv Chronic Kidney Dis 2019;26:369-75.

7. Yu F, Haas M, Glassock R, et al. Redefining lupus nephritis: clinical implications of pathophysiologic subtypes. Nat Rev Nephrol 2017;13:483-95.

8. Clement LC, Avila-Casado C, Macé C, et al. Podocytesecreted angiopoietin-like-4 mediates proteinuria in glucocorticoid-sensitive nephrotic syndrome. Nat Med 2011;17:117-22.

9. Sakhi H, Moktefi A, Bouachi K, et al. Podocyte Injury in Lupus Nephritis. J Clin Med 2019. doi: 10.3390/ jcm8091340.

10. Reiser J, von Gersdorff G, Loos M, et al. Induction of B7-1 in podocytes is associated with nephrotic syndrome. J Clin Invest 2004;113:1390-7.

11. Goldwich A, Burkard M, Olke M, et al. Podocytes are nonhematopoietic professional antigen-presenting cells. J Am Soc Nephrol 2013;24:906-16.

12. Perakis C, Arvanitis A, Sotsiou F, et al. Nephrotic syndrome caused by minimal-change disease in a patient with focal proliferative SLE nephritis (WHO III) in remission. Nephrol Dial Transplant 1998;13:467-70.

13. Yoo J, Villanueva $H$, Kaliamurthy $M$, et al. Is it coincidence or consequence for a case with antiphospholipid antibody syndrome overlapping SLE to develop an immune complex nephropathy followed by a non- immune Complex podocytopathy? Case Rep Nephrol 2018;2018:6746473.

14. Bomback AS, Markowitz GS. Lupus Podocytopathy: A Distinct Entity. Clin J Am Soc Nephrol 2016;11:547-8. 